Supporting Information:

\title{
Spherical Nanovesicles Transform into a Multitude of Nonspherical Shapes
}

\author{
Rikhia Ghosh $^{\perp}$, Vahid Satarifard ${ }^{\perp}$, Andrea Grafmüller, and Reinhard Lipowsky* \\ Theory 8 Biosystems, Max Planck Institute of Colloids and Interfaces, 14424 Pots- \\ dam, Germany
}

September 24, 2019

\section{S1. Reference state with tensionless leaflets}

As described in the main text, the spherical reference state with tensionless leaflets can be obtained by a multi-step procedure that consists of the following steps. We start from several spherical vesicles with the same volume, corresponding to the same number $N_{\mathrm{W}}^{\text {isp }}$ of enclosed water beads, and the same total number of lipids, $N_{\text {lip }}$, and vary the lipid numbers $N_{\mathrm{ol}}$ and $N_{\mathrm{il}}=N_{\text {lip }}-N_{\mathrm{ol}}$, as shown in the first column of Figure 1. Next, we compute, for each $N_{\mathrm{ol}}$-value, the leaflet tensions $\Sigma_{\mathrm{il}}$ and $\Sigma_{\mathrm{ol}}$, and use graphical extrapolation as in Figure 3 to estimate the value $N_{\mathrm{ol}}=N_{\mathrm{ol}}^{*}$, for which $\Sigma_{\mathrm{il}}=\Sigma_{\mathrm{ol}}$. We then assemble a spherical vesicle with these lipid numbers $N_{\mathrm{ol}}^{*}$ and $N_{\mathrm{il}}^{*}=N_{\text {lip }}-N_{\mathrm{ol}}^{*}$ for the same volume and the same total number of lipids. The reference state with tensionless leaflets is finally reached by slightly deflating the assembled vesicle, in this example from $\nu=1$ to $\nu=\nu_{0}=0.966$, see Table S2.

Essentially the same reference state can be obtained by the following alternative procedure. We start from the same set of spherical vesicles as before (first column of Figure 1 and Table S1) but then deflate each vesicle to $\nu=\nu_{0}<1$ in order to obtain tensionless bilayers with $\Sigma=\Sigma_{\mathrm{il}}+\Sigma_{\mathrm{ol}} \simeq 0$ (second column of Figure 1 and Table S2) The corresponding leaflet tensions are plotted in Figure S1 as a function of $N_{\text {ol }}$. Graphical extrapolation of these data then leads to the outer lipid number $N_{\mathrm{ol}}^{*}=5993$ for the reference state with tensionless leaflets. In this way, we obtain two slightly different estimates as given by $N_{\mathrm{ol}}^{*}=5963$ (Figure 3 ) and $N_{\mathrm{ol}}^{*}=5993$ (Figure S1), respectively. The small difference between these two values implies a high numerical accuracy of about $0.5 \%$.

\section{S2. Torques generated by spherical membranes}

To obtain an explicit expression for the local spontaneous curvature of the spherical vesicles considered here, we consider the first moment, $\mathcal{T}$, of the spherically symmetric stress profile $s(r)$ as given by

$$
\mathcal{T} \equiv \int_{0}^{\infty} \mathrm{d} r s(r) r=\int_{-R_{\text {mid }}}^{\infty} \mathrm{d} y s\left(R_{\text {mid }}+y\right) y+\Sigma R_{\text {mid }}
$$

where the second equality follows from a change of variable from $r$ to $y \equiv r-R_{\text {mid }}$ with the radius $R_{\text {mid }}$ of the bilayer's midsurface and the bilayer tension $\Sigma=\int \mathrm{d} r s(r)$. For a tensionless bilayer with $\Sigma=0$, the last term in Eq S1 vanishes and we are left 
with

$$
\mathcal{T}=\int_{-R_{\text {mid }}}^{\infty} \mathrm{d} y \hat{s}(y) y \quad \text { with } \quad \hat{s}(y) \equiv s\left(R_{\text {mid }}+y\right)
$$

which we interpret as the microscopic torque per unit length with respect to $y=0$. In the limit of large vesicles and, thus, large $R_{\text {mid }}$, the integral in Eq $\mathrm{S} 2$ becomes asymptotically equal to the first moment of the stress profile for planar and tensionless bilayers. [1, 2]

In Figure S2, we display the numerical values of $\mathcal{T}$ for the four spherical vesicles in Figure 1 both for $\nu=1$ and for $\nu=\nu_{0}$. Because the stress profiles $s(r)$ decay rapidly to zero as we move away from the lipid bilayer, the $r$-integration in Eq S1 was performed over the spherical shell defined by $r_{\min }=12.125 d<r<r_{\max }=35.125 d$.

On the other hand, for a lipid bilayer with bending rigidity $\kappa$ and (local) spontaneous curvature $m$, a spherical membrane segment with radius $R_{\text {mid }}$ generates the nanoscopic torque per unit length as given by $[3,4]$

$$
\mathcal{T}_{\text {nan }}=2 \kappa\left(M_{\text {mid }}-m\right) \quad \text { with } M_{\text {mid }} \equiv 1 / R_{\text {mid }} .
$$

When we identify the microscopic torque as given by Eq S2 with the nanoscopic torque in Eq S3, we obtain Eq 8 in the main text.

\section{References}

[1] B. Rozycki and R. Lipowsky. Spontaneous curvature of bilayer membranes from molecular simulations: Asymmetric lipid densities and asymmetric adsorption. J. Chem. Phys., 142:054101, 2015.

[2] M. Miettinen and R. Lipowsky. Bilayer Membranes with Frequent Flip Flops have Tensionless Leaflets. Nano Letters, 19:5011-5016, 2019.

[3] J. B. Fournier. On the stress and torque tensors in fluid membranes. Soft Matter, $3: 883-888,2007$.

[4] M. Deserno. Fluid lipid membranes: From differential geometry to curvature stresses. Chem. Phys. Lipids, 185:11-45, 2015. 


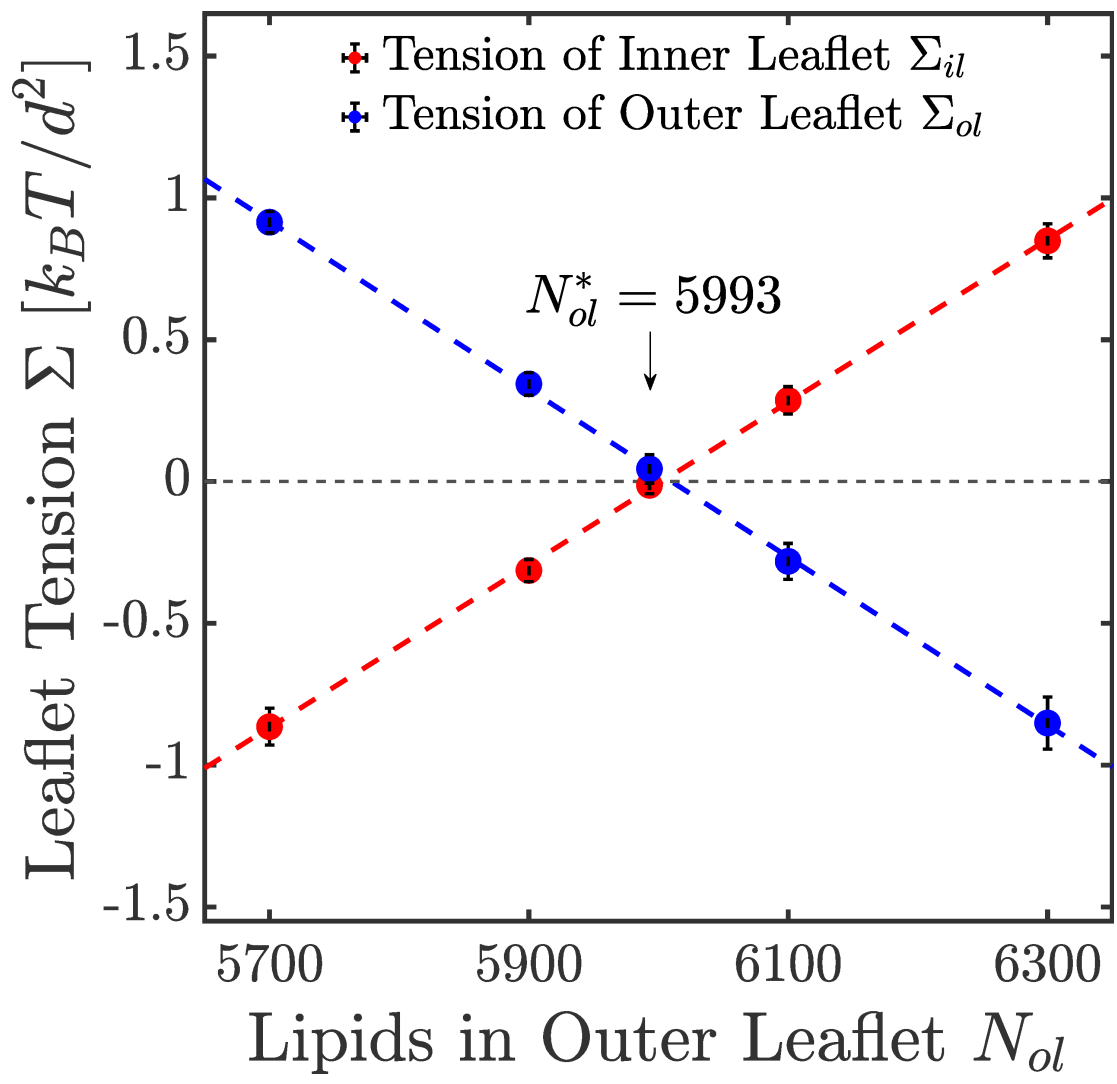

Figure S1: Leaflet tensions $\Sigma_{\mathrm{il}}$ (red) and $\Sigma_{\mathrm{ol}}$ (blue) as functions of the lipid number $N_{\text {ol }}$ in the outer leaflet for the four spherical vesicles displayed in the 2 nd column of Figure 1, corresponding to volume parameter $\nu=\nu_{0}<1$ and tensionless bilayers. The numerical parameter values are also given in Table S2. The tension $\Sigma_{\mathrm{il}}$ in the inner leaflet increases whereas the tension $\Sigma_{\mathrm{ol}}$ in the outer leaflet decreases with increasing $N_{\mathrm{ol}}$. Furthermore, both sets of data are well fitted by straight lines which cross at $N_{\mathrm{ol}}^{*}=5993$ corresponding to tensionless leaflets. 


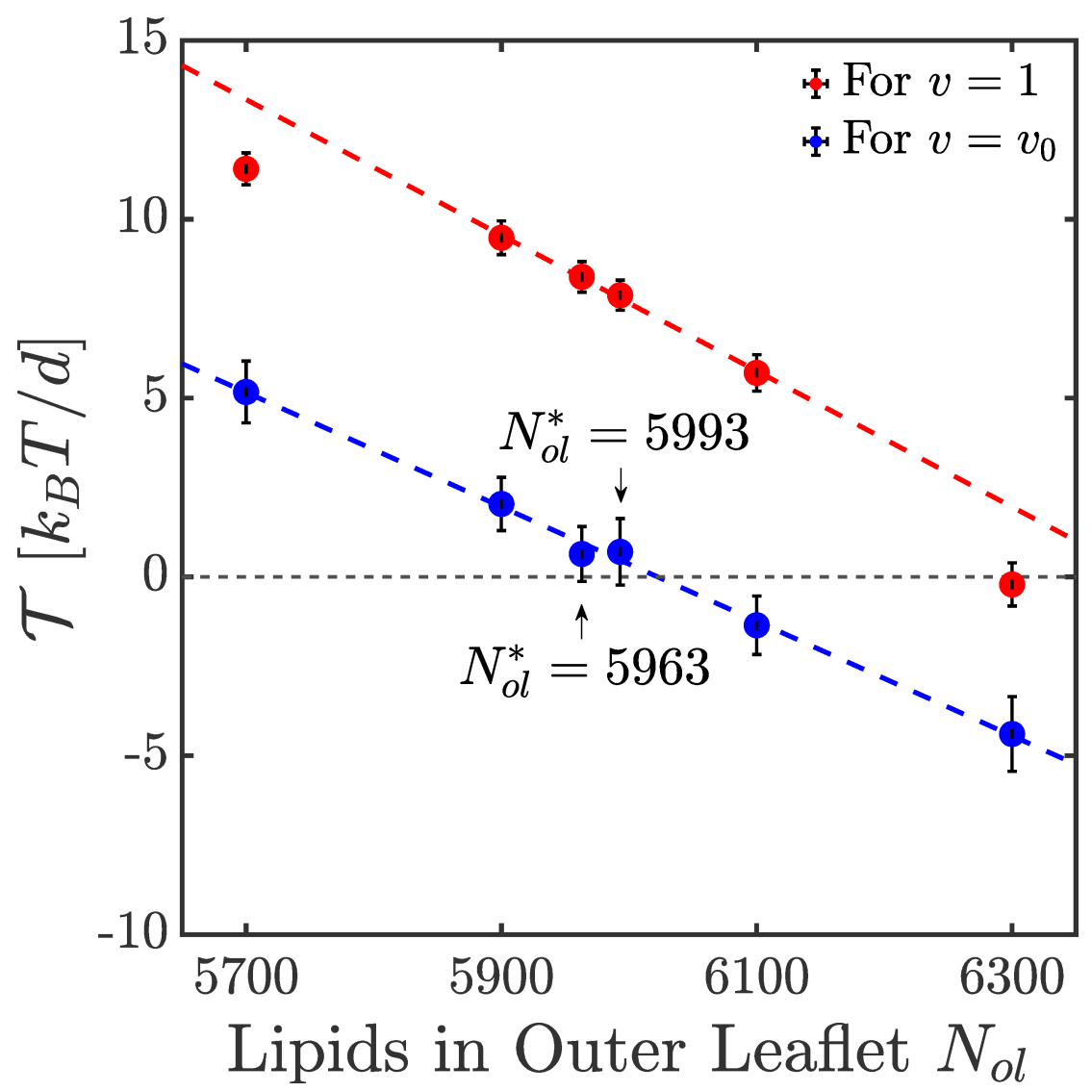

Figure S2: Dependence of first moment of stress profile, $\mathcal{T}$, on outer lipid number $N_{\mathrm{ol}}$ for the four spherical vesicles in Figure 1 and for the two spherical vesicles with $N_{\mathrm{ol}}^{*}=5963$ and $N_{\mathrm{ol}}^{*}=5993$ as determined in Figure 3 and Figure S1. Two sets of data are displayed for each vesicle, corresponding to volume parameter $\nu=1$ and $N_{\mathrm{W}}=90400$ (red) as well as to $\nu=\nu_{0}<1$ and $\Sigma \simeq 0$ (blue), see Tables S1 and S2. In contrast to the red data, the blue data are well fitted by a straight line for the whole range of $N_{\mathrm{ol}}$-values. Note that $\mathcal{T}$ does not depend on the bilayer's midsurface and, thus, has the same value for any definition of this surface. 


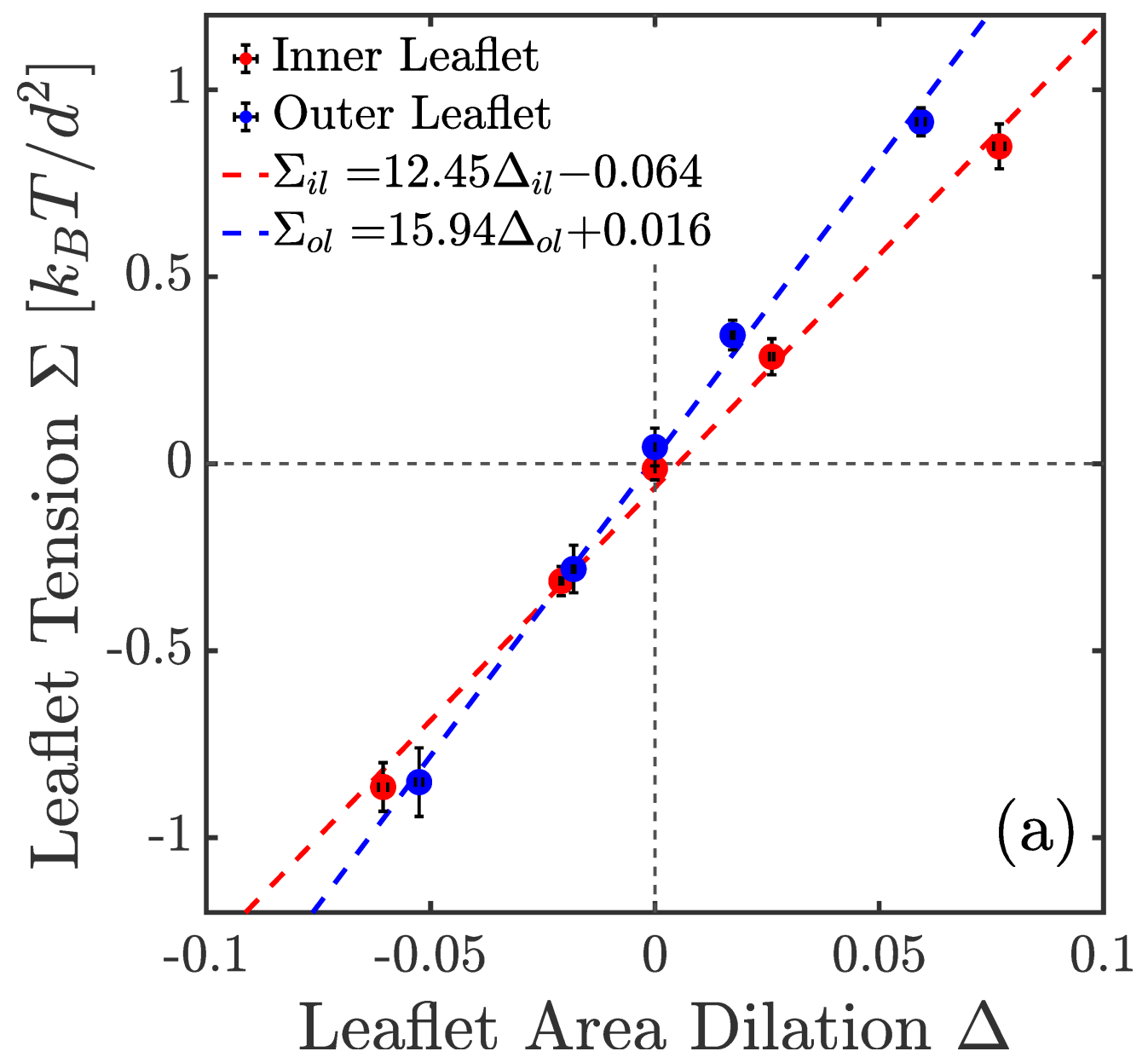

Figure S3: Leaflet tension $\Sigma_{\mathrm{il}}$ (red) and $\Sigma_{\mathrm{ol}}$ (blue) versus dimensionless area dilations $\Delta_{\mathrm{il}}$ and $\Delta_{\mathrm{ol}}$, as defined in Eqs 6 and 7 , using the midsurface radius $R_{\text {mid }}=R_{\mathrm{COM}}$ corresponding to COM, see Table S3. The straight lines represent least squares fits which determine the area compressibility moduli $K_{\mathrm{il}}=12.45 k_{\mathrm{B}} T / d^{2}$ and $K_{\mathrm{ol}}=$ $15.94 k_{\mathrm{B}} T / d^{2}$ for the inner and outer leaflets, respectively. Thus, the inner leaflet is more compressible and softer than the outer leaflet. 
Table S1: Parameter values for six spherical vesicles with volume parameter $\nu=1$ and $N_{\mathrm{W}}=90400$, corresponding to the four panels in Figure 1, Figure 4a, and the spherical vesicle with $N_{\mathrm{ol}}^{*}=5993$ determined in Figure S1. All vesicle membranes contain the same total number of lipids, $N_{\text {lip }}=N_{\mathrm{il}}+N_{\mathrm{ol}}=10100$. The midsurface radius $R_{\text {mid }}=R_{\mathrm{C}}$ of the bilayer was defined by the peak in the chain bead density $\rho_{\mathrm{C}}(r)$, see Figure 2a, corresponding to CHAIN. The midsurface radii $R_{\mathrm{il}}$ and $R_{\mathrm{ol}}$ of the inner and outer leaflets were taken to be $R_{\mathrm{il}}=\frac{1}{2}\left(R_{\mathrm{iH}}+R_{\mathrm{C}}\right)$ and $R_{\mathrm{ol}}=$ $\frac{1}{2}\left(R_{\mathrm{C}}+R_{\mathrm{oH}}\right)$ where $R_{\mathrm{iH}}$ and $R_{\mathrm{oH}}$ denote the location of the inner and outer peak of the head group density $\rho_{\mathrm{H}}(r)$, see Figure $2 \mathrm{~b}$

\begin{tabular}{r|r|r|r|r|r|r|l} 
& Figure 1a & Figure 1b & Figure 1c & Figure 1d & Figure 4a & Figure S1 & units \\
\hline$N_{\mathrm{il}}$ & 4400 & 4200 & 4000 & 3800 & 4137 & 4107 & \\
$N_{\mathrm{ol}}$ & 5700 & 5900 & 6100 & 6300 & $N_{\mathrm{ol}}^{*}=5963$ & $N_{\mathrm{ol}}^{*}=5993$ & \\
\hline$N_{\mathrm{W}}$ & 90400 & 90400 & 90400 & 90400 & 90400 & 90400 & \\
\hline$\nu$ & 1 & 1 & 1 & 1 & 1 & 1 & \\
\hline$\Sigma$ & $0.341 \pm 0.02$ & $0.376 \pm 0.02$ & $0.328 \pm 0.02$ & $0.184 \pm 0.03$ & $0.366 \pm 0.02$ & $0.36 \pm 0.02$ & $k_{\mathrm{B}} T / d^{2}$ \\
\hline$R_{\mathrm{C}}$ & $22.375 \pm 0.00$ & $22.358 \pm 0.06$ & $22.283 \pm 0.12$ & $22.133 \pm 0.05$ & $22.35 \pm 0.08$ & $22.342 \pm 0.09$ & $d$ \\
\hline$\Sigma_{\mathrm{il}}$ & $-0.706 \pm 0.02$ & $-0.048 \pm 0.04$ & $0.619 \pm 0.10$ & $1.256 \pm 0.08$ & $0.157 \pm 0.07$ & $0.259 \pm 0.07$ & $k_{\mathrm{B}} T / d^{2}$ \\
$\Sigma_{\mathrm{ol}}$ & $1.047 \pm 0.02$ & $0.424 \pm 0.04$ & $-0.292 \pm 0.09$ & $-1.073 \pm 0.09$ & $0.209 \pm 0.06$ & $0.101 \pm 0.07$ & $k_{\mathrm{B}} T / d^{2}$ \\
\hline$R_{\mathrm{il}}$ & $21.05 \pm 0.06$ & $21.013 \pm 0.04$ & $20.963 \pm 0.07$ & $20.863 \pm 0.08$ & $21.004 \pm 0.04$ & $21.004 \pm 0.04$ & $d$ \\
$R_{\mathrm{ol}}$ & $23.521 \pm 0.05$ & $23.508 \pm 0.03$ & $23.454 \pm 0.06$ & $23.363 \pm 0.08$ & $23.496 \pm 0.05$ & $23.483 \pm 0.04$ & $d$ \\
\hline$a_{\mathrm{il}}$ & $1.266 \pm 0.01$ & $1.321 \pm 0.01$ & $1.381 \pm 0.01$ & $1.439 \pm 0.01$ & $1.34 \pm 0.01$ & $1.35 \pm 0.01$ & $d^{2}$ \\
$a_{\mathrm{ol}}$ & $1.22 \pm 0.01$ & $1.177 \pm 0.00$ & $1.133 \pm 0.01$ & $1.089 \pm 0.01$ & $1.163 \pm 0.01$ & $1.156 \pm 0.00$ & $d^{2}$ \\
\hline $\mathcal{T}$ & $11.407 \pm 0.44$ & $9.482 \pm 0.47$ & $5.704 \pm 0.51$ & $-0.21 \pm 0.6$ & $8.39 \pm 0.43$ & $7.874 \pm 0.42$ & $k_{\mathrm{B}} T / d$ \\
\hline$m$ & $-0.331 \pm 0.01$ & $-0.262 \pm 0.02$ & $-0.14 \pm 0.02$ & $0.052 \pm 0.02$ & $-0.227 \pm 0.01$ & $-0.213 \pm 0.01$ & $1 / d$
\end{tabular}

Table S2: Parameter values for six spherical vesicles with volume parameter $\nu=\nu_{0}<$ 1 and tensionless bilayers, corresponding to the four panels in Figure 1, Figure 4a, and the spherical vesicle with $N_{\mathrm{ol}}^{*}=5993$ determined in Figure S1. The radii of the different midsurfaces are defined in the same way as in Table S1.

\begin{tabular}{r|r|r|r|r|r|r|l} 
& Figure 1a & Figure 1b & Figure 1c & Figure 1d & Figure 4a & Figure S1 & units \\
\hline$N_{\mathrm{il}}$ & 4400 & 4200 & 4000 & 3800 & 4137 & 4107 & \\
$N_{\mathrm{ol}}$ & 5700 & 5900 & 6100 & 6300 & $N_{\mathrm{ol}}^{*}=5963$ & $N_{\mathrm{ol}}^{*}=5993$ & \\
\hline$N_{\mathrm{W}}$ & 87350 & 87420 & 87400 & 88400 & 87360 & 87400 & \\
\hline$\nu_{0}$ & 0.966 & 0.967 & 0.967 & 0.978 & 0.966 & 0.967 & \\
\hline$\Sigma$ & $0.05 \pm 0.04$ & $0.03 \pm 0.03$ & $0.005 \pm 0.04$ & $-0.003 \pm 0.05$ & $0.01 \pm 0.03$ & $0.031 \pm 0.04$ & $k_{\mathrm{B}} T / d^{2}$ \\
\hline$R_{\mathrm{C}}$ & $22.1 \pm 0.08$ & $22.125 \pm 0.00$ & $22.017 \pm 0.13$ & $22.042 \pm 0.12$ & $22.117 \pm 0.05$ & $22.083 \pm 0.09$ & $d$ \\
\hline$\Sigma_{\mathrm{il}}$ & $-0.818 \pm 0.06$ & $-0.246 \pm 0.03$ & $0.384 \pm 0.06$ & $0.986 \pm 0.08$ & $-0.024 \pm 0.05$ & $0.064 \pm 0.04$ & $k_{\mathrm{B}} T / d^{2}$ \\
$\Sigma_{\mathrm{ol}}$ & $0.867 \pm 0.03$ & $0.277 \pm 0.05$ & $-0.38 \pm 0.07$ & $-0.989 \pm 0.10$ & $0.034 \pm 0.06$ & $-0.033 \pm 0.05$ & $k_{\mathrm{B}} T / d^{2}$ \\
\hline$R_{\mathrm{il}}$ & $20.808 \pm 0.14$ & $20.771 \pm 0.11$ & $20.688 \pm 0.15$ & $20.638 \pm 0.15$ & $20.792 \pm 0.10$ & $20.733 \pm 0.13$ & $d$ \\
$R_{\mathrm{ol}}$ & $23.342 \pm 0.16$ & $23.288 \pm 0.12$ & $23.2 \pm 0.15$ & $23.142 \pm 0.16$ & $23.3 \pm 0.08$ & $23.246 \pm 0.14$ & $d$ \\
\hline$a_{\mathrm{il}}$ & $1.237 \pm 0.02$ & $1.291 \pm 0.01$ & $1.345 \pm 0.02$ & $1.409 \pm 0.02$ & $1.313 \pm 0.01$ & $1.315 \pm 0.02$ & $d^{2}$ \\
$a_{\mathrm{ol}}$ & $1.201 \pm 0.02$ & $1.155 \pm 0.01$ & $1.109 \pm 0.01$ & $1.068 \pm 0.01$ & $1.144 \pm 0.01$ & $1.133 \pm 0.01$ & $d^{2}$ \\
\hline $\mathcal{T}$ & $5.17 \pm 0.87$ & $2.039 \pm 0.74$ & $-1.352 \pm 0.82$ & $-4.391 \pm 1.05$ & $0.641 \pm 0.77$ & $0.701 \pm 0.93$ & $k_{\mathrm{B}} T / d$ \\
\hline$m$ & $-0.117 \pm 0.03$ & $-0.02 \pm 0.02$ & $0.089 \pm 0.03$ & $0.186 \pm 0.03$ & $0.025 \pm 0.02$ & $0.023 \pm 0.03$ & $1 / d$
\end{tabular}


Table S3: Midsurface radius $R_{\text {mid }}=R_{\mathrm{C}}, R_{\mathrm{H}}$, and $R_{\mathrm{COM}}$ as obtained from CHAIN, HEAD, and COM for the five spherical vesicles in Figures 1 and $4 \mathrm{a}$ with volume parameter $\nu=\nu_{0}$ and tensionless bilayers.

\begin{tabular}{c|ccccc}
\hline Figure & $1 \mathrm{a}$ & $1 \mathrm{~b}$ & $1 \mathrm{c}$ & $1 \mathrm{~d}$ & $4 \mathrm{a}$ \\
\hline$R_{\mathrm{C}}[\mathrm{d}]$ & 22.1 & 22.1 & 22.0 & 22.0 & 22.1 \\
$R_{\mathrm{H}}[\mathrm{d}]$ & 22.1 & 21.9 & 21.9 & 21.7 & 21.9 \\
$R_{\mathrm{COM}}[\mathrm{d}]$ & 22.3 & 22.3 & 22.3 & 22.4 & 22.3 \\
\hline
\end{tabular}

Table S4: Parameter values for six spherical vesicles with volume parameter $\nu=1$ and $N_{\mathrm{W}}=90400$, corresponding to the four panels in Figure 1, Figure 4a, and the spherical vesicle with $N_{\mathrm{ol}}^{*}=5993$ determined in Figure S1. The midsurface radius $R_{\text {mid }}=R_{\mathrm{COM}}$ of the bilayer was defined by its center-of-mass, corresponding to COM. The midsurface radii $R_{\mathrm{il}}$ and $R_{\mathrm{ol}}$ of the inner and outer leaflets were taken to be $R_{\mathrm{il}}=\frac{1}{2}\left(R_{\mathrm{iH}}+R_{\mathrm{COM}}\right)$ and $R_{\mathrm{ol}}=\frac{1}{2}\left(R_{\mathrm{COM}}+R_{\mathrm{oH}}\right)$ where $R_{\mathrm{iH}}$ and $R_{\mathrm{oH}}$ denote the location of the inner and outer peak of the head group density $\rho_{\mathrm{H}}(r)$, see Figure $2 \mathrm{~b}$.

\begin{tabular}{r|r|r|r|r|r|r|l} 
& Figure 1a & Figure 1b & Figure 1c & Figure 1d & Figure 4a & Figure S1 & units \\
\hline$N_{\mathrm{il}}$ & 4400 & 4200 & 4000 & 3800 & 4137 & 4107 & \\
$N_{\mathrm{ol}}$ & 5700 & 5900 & 6100 & 6300 & $N_{\mathrm{ol}}^{*}=5963$ & $N_{\mathrm{ol}}^{*}=5993$ & \\
\hline$N_{\mathrm{W}}$ & 90400 & 90400 & 90400 & 90400 & 90400 & 90400 & \\
\hline$\nu$ & 1 & 1 & 1 & 1 & 1 & 1 & \\
\hline$\Sigma$ & $0.341 \pm 0.02$ & $0.376 \pm 0.02$ & $0.328 \pm 0.02$ & $0.184 \pm 0.03$ & $0.366 \pm 0.02$ & $0.36 \pm 0.02$ & $k_{\mathrm{B}} T / d^{2}$ \\
\hline$R_{\mathrm{COM}}$ & $22.446 \pm 0.00$ & $22.447 \pm 0.01$ & $22.445 \pm 0.01$ & $22.461 \pm 0.01$ & $22.448 \pm 0.01$ & $22.449 \pm 0.01$ & $d$ \\
\hline$\Sigma_{\mathrm{il}}$ & $-0.745 \pm 0.02$ & $-0.1 \pm 0.03$ & $0.507 \pm 0.03$ & $1.04 \pm 0.04$ & $0.097 \pm 0.03$ & $0.189 \pm 0.02$ & $k_{\mathrm{B}} T / d^{2}$ \\
$\Sigma_{\mathrm{ol}}$ & $1.086 \pm 0.02$ & $0.476 \pm 0.02$ & $-0.179 \pm 0.02$ & $-0.856 \pm 0.05$ & $0.269 \pm 0.02$ & $0.171 \pm 0.02$ & $k_{\mathrm{B}} T / d^{2}$ \\
\hline$R_{\mathrm{il}}$ & $21.086 \pm 0.06$ & $21.057 \pm 0.05$ & $21.043 \pm 0.04$ & $21.027 \pm 0.08$ & $21.053 \pm 0.05$ & $21.058 \pm 0.05$ & $d$ \\
$R_{\mathrm{ol}}$ & $23.557 \pm 0.05$ & $23.553 \pm 0.04$ & $23.535 \pm 0.03$ & $23.527 \pm 0.08$ & $23.545 \pm 0.05$ & $23.537 \pm 0.03$ & $d$ \\
\hline$a_{\mathrm{il}}$ & $1.27 \pm 0.01$ & $1.327 \pm 0.01$ & $1.391 \pm 0.01$ & $1.462 \pm 0.01$ & $1.346 \pm 0.01$ & $1.357 \pm 0.01$ & $d^{2}$ \\
$a_{\mathrm{ol}}$ & $1.223 \pm 0.01$ & $1.182 \pm 0.00$ & $1.141 \pm 0.00$ & $1.104 \pm 0.01$ & $1.168 \pm 0.01$ & $1.162 \pm 0.00$ & $d^{2}$ \\
\hline $\mathcal{T}$ & $11.407 \pm 0.44$ & $9.482 \pm 0.47$ & $5.704 \pm 0.51$ & $-0.21 \pm 0.60$ & $8.39 \pm 0.43$ & $7.874 \pm 0.42$ & $k_{\mathrm{B}} T / d$ \\
\hline$m$ & $-0.35 \pm 0.02$ & $-0.277 \pm 0.02$ & $-0.15 \pm 0.02$ & $0.052 \pm 0.02$ & $-0.241 \pm 0.01$ & $-0.226 \pm 0.01$ & $1 / d$
\end{tabular}


Table S5: Parameter values for six spherical vesicles with volume parameter $\nu=\nu_{0}<$ 1 and tensionless bilayers, corresponding to the four panels in Figure 1, Figure 4a, and the spherical vesicle with $N_{\mathrm{ol}}^{*}=5993$ determined in Figure S1. The radii of the different midsurfaces are defined in the same way as in Table S4.

\begin{tabular}{r|r|r|r|r|r|r|l} 
& Figure 1a & Figure 1b & Figure 1c & Figure 1d & Figure 4a & Figure S4a & units \\
\hline$N_{\mathrm{il}}$ & 4400 & 4200 & 4000 & 3800 & 4137 & 4107 & \\
$N_{\mathrm{ol}}$ & 5700 & 5900 & 6100 & 6300 & 5963 & 5993 & \\
\hline$N_{\mathrm{W}}$ & 87350 & 87420 & 87400 & 88400 & 87360 & 87400 & \\
\hline$\nu_{0}$ & 0.966 & 0.967 & 0.967 & 0.978 & 0.966 & 0.967 & \\
\hline$\Sigma$ & $0.05 \pm 0.04$ & $0.03 \pm 0.03$ & $0.005 \pm 0.04$ & $-0.003 \pm 0.05$ & $0.01 \pm 0.03$ & $0.031 \pm 0.04$ & $k_{\mathrm{B}} T / d^{2}$ \\
\hline$R_{\mathrm{COM}}$ & $22.315 \pm 0.02$ & $22.308 \pm 0.01$ & $22.325 \pm 0.01$ & $22.387 \pm 0.02$ & $22.306 \pm 0.01$ & $22.314 \pm 0.02$ & $d$ \\
\hline$\Sigma_{\mathrm{il}}$ & $-0.865 \pm 0.06$ & $-0.314 \pm 0.04$ & $0.286 \pm 0.05$ & $0.849 \pm 0.06$ & $-0.099 \pm 0.05$ & $-0.013 \pm 0.03$ & $k_{\mathrm{B}} T / d^{2}$ \\
$\Sigma_{\mathrm{ol}}$ & $0.914 \pm 0.04$ & $0.344 \pm 0.04$ & $-0.282 \pm 0.06$ & $-0.851 \pm 0.09$ & $0.11 \pm 0.04$ & $0.045 \pm 0.05$ & $k_{\mathrm{B}} T / d^{2}$ \\
\hline$R_{\mathrm{il}}$ & $20.916 \pm 0.12$ & $20.863 \pm 0.11$ & $20.842 \pm 0.14$ & $20.81 \pm 0.12$ & $20.886 \pm 0.11$ & $20.849 \pm 0.12$ & $d$ \\
$R_{\mathrm{ol}}$ & $23.449 \pm 0.14$ & $23.379 \pm 0.12$ & $23.354 \pm 0.14$ & $23.314 \pm 0.13$ & $23.395 \pm 0.09$ & $23.361 \pm 0.12$ & $d$ \\
\hline$a_{\mathrm{il}}$ & $1.249 \pm 0.01$ & $1.302 \pm 0.01$ & $1.365 \pm 0.02$ & $1.432 \pm 0.02$ & $1.325 \pm 0.01$ & $1.33 \pm 0.02$ & $d^{2}$ \\
$a_{\mathrm{ol}}$ & $1.212 \pm 0.01$ & $1.164 \pm 0.01$ & $1.124 \pm 0.01$ & $1.084 \pm 0.01$ & $1.153 \pm 0.01$ & $1.144 \pm 0.01$ & $d^{2}$ \\
\hline $\mathcal{T}$ & $5.17 \pm 0.87$ & $2.039 \pm 0.74$ & $-1.352 \pm 0.82$ & $-4.391 \pm 1.05$ & $0.641 \pm 0.77$ & $0.701 \pm 0.93$ & $k_{\mathrm{B}} T / d$ \\
\hline$m$ & $-0.125 \pm 0.03$ & $-0.023 \pm 0.02$ & $0.09 \pm 0.03$ & $0.193 \pm 0.04$ & $0.023 \pm 0.03$ & $0.021 \pm 0.03$ & $1 / d$
\end{tabular}




\section{Supporting Information}

\section{Spherical Nanovesicles Transform into a Multitude of Nonspherical Shapes}

Rikhia Ghosh ${ }^{\perp}$, Vahid Satarifard ${ }^{\perp}$, Andrea Grafmüller, and Reinhard Lipowsky*

Theory and Bio-Systems, Max Planck Institute of Colloids and Interfaces, Science Park Golm, 14424 Potsdam, Germany

September 24, 2019

\section{Movie Captions}

Movie S1. Shape transformation of a nanovesicle with $N_{\mathrm{il}}=4400$ and $N_{\mathrm{ol}}=5700$ lipid molecules in the inner and outer leaflet of the vesicle membrane. The initial vesicle at time $t=0 \mu \mathrm{s}$ has a spherical shape with volume parameter $\nu=1$ corresponding to $N_{\mathrm{W}}^{\text {isp }}=90400$ enclosed water beads. The video displays four successive deflation steps from volume $\nu=1.0$ to $\nu=0.95$, from $\nu=0.95$ to $\nu=0.9$, from $\nu=0.9$ to $\nu=0.85$, and from $\nu=0.85$ to $\nu=0.8$, with a total run time of $138.5 \mu \mathrm{s}$. The final shape is a stomatocyte, compare Figure 1a in the main text.

Movie S2. Shape transformation of a nanovesicle with $N_{\mathrm{il}}=3800$ and $N_{\mathrm{ol}}=6300$ lipid molecules in the inner and outer leaflet of the vesicle membrane. The initial vesicle at time $t=0 \mu \mathrm{s}$ has a spherical shape with volume parameter $\nu=1$ corresponding to $N_{\mathrm{W}}^{\text {isp }}=90400$ enclosed water beads. The video displays a single deflation step from $\nu=1$ to $\nu=0.8$, with a total runtime of $50 \mu \mathrm{s}$. The final shape is a dumbbell with a closed membrane neck, compare Figure 1d in the main text. 